\title{
Robust Chance-Constrained Secure Transmission for Cognitive Satellite-Terrestrial Networks
}

\author{
Bin Li, Zesong Fei, Senior Member, IEEE, Zheng Chu, Member, IEEE, Fuhui Zhou, Member, IEEE, \\ Kai-Kit Wong, Fellow, IEEE, and Pei Xiao, Senior Member, IEEE
}

\begin{abstract}
Cognitive satellite-terrestrial networks (CSTNs) have been recognized as a promising network architecture for addressing spectrum scarcity problem in next-generation communication networks. In this paper, we investigate the secure transmission for CSTNs where the terrestrial base station (BS) serving as a green interference resource is introduced to enhance the security of the satellite link. Adopting a stochastic model for the channel state information (CSI) uncertainty, we propose a secure and robust beamforming framework to minimize the transmit power, while satisfying a range of outage (probabilistic) constraints concerning the signal-to-interference-plus-noise ratio (SINR) recorded at the satellite user and the terrestrial user, the leakage-SINR recorded at the eavesdropper, as well as the interference power recorded at the satellite user. The resulting robust optimization problem is highly intractable and the key observation is that the highly intractable probability constraints can be equivalently reformulated as the deterministic versions with Gaussian statistics. In this regard, we develop two robust reformulation methods, namely $\mathcal{S}$-Procedure and Bernstein-type inequality restriction techniques, to obtain a safe approximate solution. In the meantime, the computational complexities of the proposed schemes are analyzed. Finally, the effectiveness of the proposed schemes are demonstrated by numerical results with different system parameters.
\end{abstract}

Index Terms-Satellite-terrestrial networks, physical-layer security, robust beamforming, outage probability, power minimization.

\section{INTRODUCTION}

A $\mathrm{S}$ broadcast applications and multimedia services are becoming increasingly popular, cooperative satellite and terrestrial cell network has been emerged as a future heterogeneous network, which is capable of increasing coverage range and conveying rich multimedia services (e.g., video on demand (VoD) streaming and TV broadcasting, etc.) to users

Copyright (c) 2015 IEEE. Personal use of this material is permitted. However, permission to use this material for any other purposes must be obtained from the IEEE by sending a request to pubs-permissions@ieee.org.

B. Li and Z. Fei are with the School of Information and Electronics, Beijing Institute of Technology, Beijing 100081, China (e-mail: libin_sun@bit.edu.cn; feizesong@bit.edu.cn).

Z. Chu and Pei Xiao are with the 5G Innovation Center (5GIC), Institute of Communication Systems (ICS), University of Surrey, Guildford, GU2 7XH, U.K. (e-mail: andrew.chuzheng7@gmail.com; p.xiao@surrey.ac.uk).

F. Zhou is with the Department of Electrical and Computer Engineering, Utah State University, Logan, UT, USA, and is also with the School of Information Engineering and Post-Doctoral Research Station of Environmental Science and Engineering, Nanchang University, Nanchang 330031, China (email: zhoufuhui1989@163.com).

K.-K. Wong is with the Department of Electronic and Electrical Engineering, University College London, London WC1E 6BT, U.K. (e-mail: kaikit.wong@ucl.ac.uk). anywhere [1], [2]. However, the exponentially growing demand for multimedia contents may result in the spectrum scarcity. To address this issue, cognitive radio has recently been applied into the satellite-terrestrial network to improve the utilization of radio spectrum resource, which is termed as cognitive satelliteterrestrial network (CSTN) [3], [4]. This allows the satellite network and the terrestrial cell network operating in the same frequency band, playing a vital role in the development and full realization of $5 \mathrm{G}$ networks.

Security is a critical concern faced by satellite communications [5] since the wireless information intended for the destination devices are also obtained by the non-intended third parties (i.e., eavesdroppers (Eves)) in the same beam due to the openness of wireless links. In order to provide a high level of information security, appropriate signal processing and communication technologies need to be invoked to guarantee the secure communication and the link qualities. Conventional strategies to secure communication for preventing unauthorized reception by Eves rely on cryptographic encryption implemented in higher layer. However, the encryption based methods entail a relatively high computation burden due to the required key distribution and service management with the explosive growth of wireless devices. As a complementary solution, physical-layer security has attracted significant attention for safeguarding wireless networks. In this line of research, physical-layer security for the multiple-input singleoutput channels [6], multiple-input multiple-output channels [7], relay channels [8], interference channels [9] and cognitive channels [10] have been well pursued. Additionally, the concept of directional modulation is recently exploited to enhance the security from a practical implementation perspective [11]. In this paper, we focus on physical-layer security in CSTNs by taking advantage of the time-variability of wireless medium to achieve secure transmission against eavesdropping.

\section{A. Related Works and Motivation}

Recent years have witnessed the research advancement in the field of hybrid/integrated satellite-terrestrial cooperative networks for future multimedia services (e.g., see [1], [12]). For instance, the hybrid satellite-terrestrial cooperative networks were investigated in [13]-[17] based on Digital Video BroadcastSatellite Handheld (DVB-SH) standard. In this situation, many researchers have analyzed the performance of hybrid satelliteterrestrial networks in single antenna [16], [18] and multiantenna [17] scenarios, respectively. It is foreseeable that the 
number of connected devices will exceed 20 billion until 2020, which requires extra spectrum resources. In this regard, the idea of combining cognitive radio into satellite-terrestrial networks has been presented in the literature [4], [19]-[21] with the aim of enhancing the spectrum utilization by sharing the same spectrum between the wireless terrestrial and satellite networks. It is envisaged that the C-band (4-8 GHz) and S-band (2-4 GHz) are to be shared between the terrestrial network and satellite network. Also, the above $10 \mathrm{GHz} \mathrm{Ka}$ band is the most promising candidate for cognitive satellite communications with significant practical applications [22].

A number of research efforts have been dedicated in studying CSTNs. Specifically, [20] provided possible cognitive coexistence scenarios between the satellite and terrestrial networks, and discussed several cognitive techniques as enablers. [4] studied an optimal power allocation mechanism in the novel architecture, and [21] presented the cognitive exploitation schemes for both the forward- and return-links devoting the improvement of system performance. Considering the real-time applications in the CSTNs, the authors in [23] maximized the delay-limited capacity and outage capacity of the satellite link by designing two power control schemes. In [24], the outage probability expression of the terrestrial secondary user (SU) was derived in a closed-form while satisfying the interference constraint to the satellite primary user (PU) imposed by wireless terrestrial network.

In recent years, the capability of enhancing the privacy and security of satellite communications is also an attractive benefit brought by physical-layer security compared to cryptographic encryption [25]. In the seminal work of [26], Lei et al. studied the security oriented beamforming and power control in a multibeam satellite communication in order to minimize the transmit power, while maintaining individual secrecy rate constraint. Subsequently, [27] proposed the optimal strategy design to improve the security of multibeam satellite communication, and the artificial noise was explored as an additional degreeof-freedom to protect against Eves. To circumvent the masking effect from the satellite to the terrestrial terminals, the authors of [28] investigated the secure transmission with the assistance of a relay for a hybrid satellite-terrestrial network. The framework of physical-layer security to enhance secrecy performance in CSTNs was first established in [3], where the authors derived the tractable expressions of the secrecy outage probability of satellite user (i.e., PU). Meanwhile, a joint beamforming design at satellite and terrestrial BS for a CSTN was proposed in [29] so as to maximize the secrecy rate of the PU. Although the aforementioned works have provided a solid understanding of physical-layer security in the satellite communications, the research on the secrecy communication for CSTNs is still in its infancy.

The channel state information (CSI) availability is one of the utmost important issues in implementing the secure transmission design. In practical instances, it is very challenging to obtain accurate CSI at each communication node due to channel quantization errors, feedback delay and dynamics of wireless channels, especially in CSTNs where the information exchange between the satellite and terrestrial networks is limited. Therefore, robust design has aroused great interests due to the ability of offering performance guarantees for optimization problems with uncertainty [3], [14]. In general, there are two classes of models to characterize imperfect CSI: the deterministic (worst-case) and stochastic uncertainty models. Taking the deterministic uncertainty model into account, a growing body of robust secure transmission for satellite communications has been studied in [26], [27], in which the CSI uncertainty is bounded by possible values. We note that the worst-case setting characterized by deterministic uncertainty model infrequently occurs and may not reflect practical channels accurately. While in contrast, the stochastic uncertainty model is a better choice for dealing with the estimated errors which are often modeled as Gaussian random variables [30]. It has been shown in [31] that the probabilistic design using a stochastic uncertainty model could guarantee a certain chance of successful quality of service (QoS) deliveries (1-outage probability\%), which was a very important performance metric in satellite communications.

In CSTNs, the interference from the terrestrial BS is beneficial to combat the malicious eavesdropping attack but is harmful to the satellite user (i.e., PU) in the satellite network. As a result, the terrestrial network has to properly design its transmit beamforming vector by considering the interference to the PU and Eve. However, prior works paid attention to the robust secure communication in the satellite network solely, there is no work focusing on the stochastic secure beamforming design with CSI uncertainty for CSTNs, which is the emphasis of this paper.

\section{B. Approaches and Contributions}

This paper studies the physical-layer security for CSTNs consisting of a satellite network and a terrestrial network. To realize the green communication target, we consider the robust design of power minimization transmission strategies in a secure CSTN by incorporating channel uncertainties. Unlike [3], from a more practical point of view, the probabilistic CSI error model is employed in our work to address the robust beamforming design problem, while maintaining the SINR outage constraints ${ }^{1}$ for PU, Eve and SU. The detailed contributions are summarized as follows.

- We investigate the problem of downlink beamforming for secure CSTN by considering the practical scenario of imperfect CSI regarding the PU's, Eve's and SU's links, where the interference from the terrestrial network can be utilized for confounding the Eve. To the best knowledge of the authors, the robust problem of interference-aware resource allocation in CSTN has not been addressed in the literature.

- We propose a stochastic beamforming framework to minimize the transmit power while ensuring the outage QoS requirements, i.e., the minimum SINR target at PU, the maximum tolerance leakage-SINR target at Eve, the minimum transmission SINR target at SU and the interference

\footnotetext{
${ }^{1}$ Note that the terms outage constraint and probabilistic constraint are used interchangeably in this paper for convenience.
} 
power constraint to PU. Under this framework, the CSI is characterized by a probabilistic model. Such problem is non-convex owing to lack of the closed-form expressions associated with the outage constraints, thus we develop two conservative approximation methods, i.e., $\mathcal{S}$-Procedure and Bernstein-type inequality.

- With the aid of the two kinds of mathematical tools, we reformulate the original problem into an easier problem respectively, which has finite constraints. Then, we apply the semidefinite relaxation (SDR) and construct the solvable semidefinite programming (SDP) problems, which result in safe $^{2}$ approximate solutions.

- Finally, we show that the solutions of the relaxed SDP problems always yield rank-one, which indicate that the relaxations are tight. Furthermore, the computational complexities for the proposed schemes are evaluated.

The remainder of this paper is structured as follows. In Section II, we present the network model and the channel model. In Section III, we formulate the corresponding outage constrained optimization problem using stochastic CSI model. In Section IV, we propose two robust schemes to address the original complex optimization problem. Numerical results are provided in Section V. Finally, the conclusions of this paper are offered in Section VI.

Notations: Vectors and matrices are denoted by bold lower case and upper case letters. $\mathbf{A}^{H}, \operatorname{rank}(\mathbf{A})$ and $\operatorname{Tr}(\mathbf{A})$ indicate the conjugate transpose, rank and trace of the matrix $\mathbf{A}$, respectively. The expectation of a random variable is represented by $\mathbb{E}\{\cdot\}$ and the Euclidean norm of a vector is represented by $\|\cdot\|$. Random vector $\mathbf{x} \sim \mathcal{C N}(\boldsymbol{\mu}, \mathbf{\Phi})$ follows the distribution of a complex Gaussian with mean $\boldsymbol{\mu}$ and covariance matrix $\boldsymbol{\Phi}$. By $\mathbf{X} \succeq \mathbf{0}$, we mean that $\mathbf{X}$ is a positive semidefinite matrix. The operator $\operatorname{Pr}\{\cdot\}$ denotes the probability measure, $\operatorname{Re}\{\cdot\}$ denotes the real part of the scalar, and $[\mathbf{R}]_{m, n}$ denotes $(m, n)$-th component of matrix $\mathbf{R}$.

\section{SYSTEM MODEL}

Consider a downlink CSTN as depicted Fig. 1, where the satellite network coexists the terrestrial network sharing the same radio spectrum resource. Specifically, the satellite downlink corresponds to the primary network and the terrestrial downlink corresponds to the secondary network. In the primary network, the geostationary orbit (GEO) satellite (SAT) with single antenna (a.k.a. feed) sends confidential message to the fixed satellite terminal (i.e., PU) in the presence of an Eve attempting to overhear the satellite information signal in the same beam. In the secondary network, the terrestrial BS is assumed to has $N_{t}$ antennas communicating with a terrestrial user $^{3}$ (i.e., SU) for simplicity. We assume that the PU, Eve and SU each has a single antenna. In this considered CSTN, the co-channel interference is taken into account. In particular, the transmit signal from the terrestrial BS, as a friendly interference

\footnotetext{
"Safe" indicates that the feasible set composed by the approximated constraint is a subset of the original feasible set.

${ }^{3}$ We note that the considered system model can be extended to include multiple SUs at the expense of a more involved notation.
}

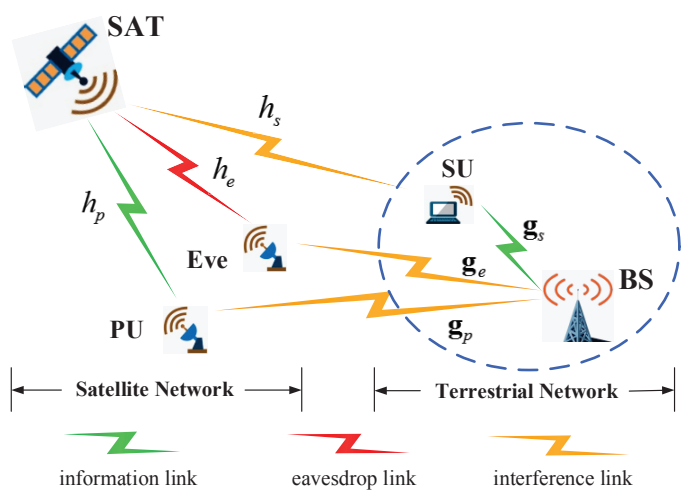

Fig. 1. System model of cognitive satellite-terrestrial networks.

resource, can be introduced into the satellite link for improving communication security of the PU.

Of particular note, the terrestrial network only knows the stochastic CSI of PU, Eve, SU, which is a more practical assumption that distinguishes our work from state-of-the-art. The considered scenario may find wide applications in future 5G wireless communication systems (such as those for multimedia services). Furthermore, since the satellite channel and the terrestrial channel are inherently different, the physical-layer security in CSTNs is more challenging. In what follows, we will discuss the detailed channel models.

\section{A. Satellite Channel Model}

It is well known that the radiowave propagation over the air is impaired by various atmospheric effects, such as rain, fog, poor angle of inclination, etc. However, the satellite links operate at high frequency bands, the atmospheric attenuation will be the major impairment for propagations channels. For practical purposes, the satellite link is modeled by composite fading distribution for describing the statistical properties of the signal envelope accurately. Currently, the Shadowed-Rician fading model has been widely-adopted in the literature [3], [24], [32]. Based on this model, the corresponding channel fading coefficient is given by

$$
\tilde{h}_{i}=A \exp \left(j \psi_{i}\right)+Z \exp \left(j \phi_{i}\right),
$$

where $\tilde{h}_{i}(i \in\{p, e, s\})$ is the channel coefficient between SAT and the corresponding terminal $i$, which includes the scattering and the line-of-sight (LOS) components. $\psi_{i} \in[0,2 \pi)$ represents the stationary random phase and $\phi_{i}$ represents the deterministic phase of the LOS component. $A$ and $Z$ denote the amplitudes of the scattering and the LOS components, which are independent stationary random processes following Rayleigh and Nakagami$m$ distributions, respectively. Specifically, the Shadowed-Rician fading distribution can be represented as $\tilde{h}_{i}=\left(b_{i}, m_{i}, \Omega_{i}\right)$, with $2 b_{i}$ being the average power of the scatter component, $\Omega_{i}$ the average power of the LOS component and $0 \leq m_{i} \leq \infty$ the Nakagami fading parameter.

On another front, for the large distance from the SAT to satellite terminals, free-space path loss is an immediate obstacle 
in satellite channels. According to [33], the free-space path loss coefficient can be written as

$$
C_{L}=\left(\frac{\lambda}{4 \pi}\right)^{2} \frac{1}{d_{0}^{2}+d_{h}^{2}},
$$

where $\lambda$ stands for the carrier wavelength, $d_{0}$ denotes the distance of the center of the satellite coverage area from the center of the central beam, and $d_{h}=35786 \mathrm{~km}$ accounts for the height of SAT.

In addition, the beam gain is determined by the SAT antenna pattern and the position of a user. Given a user's position within the satellite spot beam coverage area, the beam gain factor can be expressed as [27], [34]

$$
b\left(\varphi_{i}\right)=b_{\max }\left(\frac{J_{1}\left(u_{i}\right)}{2 u_{i}}+36 \frac{J_{3}\left(u_{i}\right)}{u_{i}^{3}}\right)^{2},
$$

with

$$
u_{i}=2.07123 \frac{\sin \varphi_{i}}{\sin \left(\varphi_{3 \mathrm{~dB}}\right)_{i}},
$$

where $b\left(\varphi_{i}\right)$ depends on user $i$ 's location. $b_{\max }$ is the maximal satellite antenna gain, $\varphi_{3 \mathrm{~dB}}$ represents the $3-\mathrm{dB}$ angle, $\varphi_{i}$ represents the angle between the corresponding user $i$ and the beam center from the SAT. $J_{1}(\cdot)$ and $J_{3}(\cdot)$ correspond to the first-kind Bessel functions of order 1 and 3.

Integrating the above three main factors, for any given satellite link, the entire satellite channel can be modeled as

$$
h_{i}=\sqrt{b\left(\varphi_{i}\right)} \tilde{h}_{i}, \forall i \in\{p, e, s\} .
$$

\section{B. Terrestrial Channel Model}

For the terrestrial cellular network, the terrestrial BS and user terminals may not be in close proximity to each other. According to the channel model introduced by [35], we assume that the terrestrial links go through the correlated Rayleigh fading without loss of generality. Using the commonly adopted Kronecker model, the terrestrial channel vector is modeled as

$$
\mathbf{g}_{i}=\mathbf{R}_{i}^{\frac{1}{2}} \tilde{\mathbf{g}}_{i}, \forall i \in\{p, e, s\},
$$

where $\tilde{\mathbf{g}}_{i} \in \mathbb{C}^{N_{s} \times 1}(i \in\{p, e, s\})$ denotes the channel gain between the terrestrial BS and user $i$, which follows Rayleigh fading. $\mathbf{R}_{i}$ is the corresponding correlation matrix. Following the study of [3], the uniform linear antenna (ULA) array is assumed to be adopted at the terrestrial BS, and the correlation matrix $\mathbf{R}_{i},(i=\{p, e, s\})$ with the $(m, n)$-th element can be computes as

$$
\left[\mathbf{R}_{i}\right]_{m, n} \approx \frac{1}{2 \pi} \int_{0}^{2 \pi} \exp \left[-j 2 \pi(m-n) \Delta \theta_{i} \frac{d}{\lambda} \sin \theta_{i}\right] d \theta,
$$

where $\theta_{i}$ corresponds to the angle-of departure (AOD), $\Delta \theta_{i}$ is the angle spread and $d$ is the distance between the two adjacent antennas.

\section{Problem Formulation}

Let $x_{p}$ and $x_{s}$ be the signals transmitted by the SAT and the terrestrial BS, with unit power $\mathbb{E}\left\{\left|x_{p}\right|^{2}\right\}=1$ and $\mathbb{E}\left\{\left|x_{s}\right|^{2}\right\}=$ 1 respectively without loss of generality. The linear transmit beamforming is performed at the terrestrial BS to protect the PU from eavesdropping, then the received signal $y_{p}$ at PU can be formulated as

$$
y_{p}=\sqrt{P_{p}} h_{p} x_{p}+\mathbf{g}_{p}^{H} \mathbf{w} x_{s}+n_{p},
$$

and $y_{e}$ at Eve can be formulated as

$$
y_{e}=\sqrt{P_{p}} h_{e} x_{p}+\mathbf{g}_{e}^{H} \mathbf{w} x_{s}+n_{e},
$$

and $y_{s}$ at $\mathrm{SU}$ can be formulated as

$$
y_{s}=\mathbf{g}_{s}^{H} \mathbf{w} x_{s}+\sqrt{P_{p}} h_{s} x_{p}+n_{s},
$$

where $P_{p}$ denotes the transmit power of the SAT and $\mathbf{w} \in$ $\mathbb{C}^{N_{t} \times 1}$ is the beamforming vector. $n_{i} \sim \mathcal{C N}\left(0, \sigma_{i}^{2}\right)(i \in$ $\{p, e, s\})$ denotes additive Gaussian noise at the corresponding receiver $i$, where $\sigma_{i}^{2}$ denotes the corresponding background noise power.

Assuming all the receiving nodes apply single user detection, according to (8) and (9), the achievable SINRs at PU and Eve can be expressed, respectively, as

$$
\begin{aligned}
\operatorname{SINR}_{p} & =\frac{P_{p}\left|h_{p}\right|^{2}}{\left|\mathbf{g}_{p}^{H} \mathbf{w}\right|^{2}+\sigma_{p}^{2}}, \\
\operatorname{SINR}_{e} & =\frac{P_{p}\left|h_{e}\right|^{2}}{\left|\mathbf{g}_{e}^{H} \mathbf{w}\right|^{2}+\sigma_{e}^{2}} .
\end{aligned}
$$

It can be seen from (11) and (12) that the interference from the terrestrial BS affects both $\mathrm{SINR}_{p}$ and $\mathrm{SINR}_{e}$. If we implement transmit beamforming design at the terrestrial BS to deteriorate the output SINR of Eve, the security of PU can be improved. To this end, beamforming vector $\mathbf{w}$ should be properly managed in accordance with the channel condition such that the transmit signal from the terrestrial BS does not severely interfere with the PU.

Similarly, from (10), the achievable SINR at SU can be expressed as

$$
\operatorname{SINR}_{s}=\frac{\left|\mathbf{g}_{s}^{H} \mathbf{w}\right|^{2}}{P_{p}\left|h_{s}\right|^{2}+\sigma_{s}^{2}}
$$

In practical communication systems, the channel knowledge of PU and Eve is unavailable at the terrestrial BS since the PU and Eve are within the satellite network ${ }^{4}$ [36]. Meanwhile, we assume that the CSI of SU is also unavailable to the terrestrial BS due to estimated and feedback errors. In addition, we assume that the channel knowledge of satellite links is available at the SAT, which is possible because the satellite terminals can be monitored and can use feedback/training data. However, the CSI of satellite terminals may not be available at the SAT due to the large distance, we will consider this case in our future work. In this setup, we adopt a stochastic model [30], [37] to characterize

\footnotetext{
${ }^{4}$ This is because for transmitter-receiver pairs in different networks, it is very hard for the terrestrial BS to know the precise CSI of the terrestrial BS-PU link $\mathbf{g}_{p}$ and the terrestrial BS-Eve link $\mathbf{g}_{e}$.
} 
the CSI imperfection, thus the channel vectors can be modeled, respectively, as

$$
\mathbf{g}_{p}=\hat{\mathbf{g}}_{p}+\Delta \mathbf{g}_{p}, \mathbf{g}_{e}=\hat{\mathbf{g}}_{e}+\Delta \mathbf{g}_{e}, \mathbf{g}_{s}=\hat{\mathbf{g}}_{s}+\Delta \mathbf{g}_{s},
$$

where $\hat{\mathrm{g}}_{p}, \hat{\mathrm{g}}_{e}$ and $\hat{\mathrm{g}}_{s}$ denote the imperfect channel estimate vectors, which are known to the terrestrial BS. And $\Delta \mathbf{g}_{p}, \Delta \mathbf{g}_{e}$ and $\Delta \mathbf{g}_{s}$ denote the corresponding channel errors/uncertainties, which tend to be Gaussian random variables with zero-mean and covariance regarding $\Delta \mathbf{g}_{p} \sim \mathcal{C N}\left(\mathbf{0}, \boldsymbol{\Omega}_{p}\right), \Delta \mathbf{g}_{e} \sim \mathcal{C N}\left(\mathbf{0}, \boldsymbol{\Omega}_{e}\right)$, $\Delta \mathbf{g}_{s} \sim \mathcal{C N}\left(\mathbf{0}, \boldsymbol{\Omega}_{s}\right)$, where $\boldsymbol{\Omega}_{p} \succeq \mathbf{0}, \boldsymbol{\Omega}_{e} \succeq \mathbf{0}$, and $\boldsymbol{\Omega}_{s} \succeq \mathbf{0}$ stand for the covariance matrices corresponding channel estimation errors.

Correspondingly, the CSI error vectors are rewritten as

$$
\begin{aligned}
\Delta \mathbf{g}_{p} & =\boldsymbol{\Omega}_{p}^{1 / 2} \mathbf{r}_{p}, \boldsymbol{\Omega}_{p}=\boldsymbol{\Omega}_{p}^{1 / 2} \boldsymbol{\Omega}_{p}^{1 / 2}, \\
\Delta \mathbf{g}_{e} & =\boldsymbol{\Omega}_{e}^{1 / 2} \mathbf{r}_{e}, \boldsymbol{\Omega}_{e}=\boldsymbol{\Omega}_{e}^{1 / 2} \boldsymbol{\Omega}_{e}^{1 / 2}, \\
\Delta \mathbf{g}_{s} & =\boldsymbol{\Omega}_{s}^{1 / 2} \mathbf{r}_{s}, \boldsymbol{\Omega}_{s}=\boldsymbol{\Omega}_{s}^{1 / 2} \boldsymbol{\Omega}_{s}^{1 / 2},
\end{aligned}
$$

where we have $\mathbf{r}_{p} \sim \mathcal{C N}\left(\mathbf{0}, \mathbf{I}_{N_{t}}\right), \mathbf{r}_{e} \sim \mathcal{C N}\left(\mathbf{0}, \mathbf{I}_{N_{t}}\right)$ and $\mathbf{r}_{s} \sim$ $\mathcal{C N}\left(\mathbf{0}, \mathbf{I}_{N_{t}}\right)$.

In this considered scenario, the SU and PU operate on the same spectrum resource. However, the PU is licensed user and hence the terrestrial $\mathrm{BS}$ is required to guarantee the QoS of the PU via a proper transmit beamforming design. To guarantee the green and secure communications, we aim to minimize the power consumption of the terrestrial BS by designing the beamforming vector $\mathbf{w}$ under the SINR outage limit for the PU, the leakage-SINR outage limit for the Eve, the SINR outage limit for the SU, and the interference outage limit for the PU. Therefore, the robust optimization problem can be formulated as

$$
\begin{array}{ll} 
& \min _{\mathbf{w}}\|\mathbf{w}\|^{2} \\
\text { s.t. } & \operatorname{Pr}\left\{\operatorname{SINR}_{p} \geq \gamma_{p}\right\} \geq 1-\rho_{p}, \\
& \operatorname{Pr}\left\{\operatorname{SINR}_{e} \leq \gamma_{e}\right\} \geq 1-\rho_{e}, \\
& \operatorname{Pr}\left\{\operatorname{SINR}_{s} \geq \gamma_{s}\right\} \geq 1-\rho_{s}, \\
& \operatorname{Pr}\left\{\left|\mathbf{g}_{p}^{H} \mathbf{w}\right|^{2} \leq \gamma_{t h}\right\} \geq 1-\rho_{t h},
\end{array}
$$

where $\gamma_{p}>0$ represents the prescribed SINR target of the PU for information decoding, $\gamma_{e}>0$ denotes the maximum tolerable SINR of the Eve for eavesdropping, $\gamma_{s}>0$ and $\gamma_{t h}>0$ denote the minimum SINR threshold of the SU for guaranteeing the reliable communication and the maximum tolerable interference constraint of the PU; $\rho_{p} \in(0,1], \rho_{e} \in(0,1]$, $\rho_{s} \in(0,1]$ and $\rho_{t h} \in(0,1]$ stand for the prescribed maximal outage probability of the SINR recorded at the PU, of the SINR recorded at the Eve, of the SINR at the SU, as well as of the interference recorded at the PU, respectively. Furthermore, the constraint $(18 \mathrm{~b})$ represents the probability of the minimum required received SINR of the PU $\gamma_{p}$ being no less than $\left(1-\rho_{p}\right) \times 100 \%$; the constraint (18c) represents the probability of the maximum tolerable received SINR of the Eve $\gamma_{e}$ being no less than $\left(1-\rho_{e}\right) \times 100 \%$; the constraint (18d) represents the probability of the minimum required received SINR of the SU $\gamma_{s}$ being no less than $\left(1-\rho_{s}\right) \times 100 \%$; the constraint (18e) represents the probability of the maximum tolerable interference threshold of the PU $\gamma_{t h}$ being no less than $\left(1-\rho_{t h}\right) \times 100 \%$.

In problem (18), the minimum guaranteed SINR constraint of PU and the maximum tolerable SINR constraint of Eve can be reformulated as secret rate constraint [30], which due to the fact that the secret rate monotonically increases with the SINR at PU but decreases with that at Eve. For given $\gamma_{p}>0$ and $\gamma_{e}>0$, the secret rate of PU can be expressed as $R_{\mathrm{sec}}=\left[\log _{2}\left(1+\mathrm{SINR}_{p}\right)-\log _{2}\left(1+\mathrm{SINR}_{e}\right)\right] \geq$ $\left[\log _{2}\left(1+\gamma_{p}\right)-\log _{2}\left(1+\gamma_{e}\right)\right]$. As a result, we know from the constraints (18b) and (18c) that, by adjusting the values of given SINR targets, the lower bound of secret rate $R_{\mathrm{sec}}$ can be guaranteed by solving problem (18).

Remark 1: In fact, the secondary link can access the spectrum licensed to PU under the condition that the transmission security of the primary link is ensured. This model has been well justified in the secure resource allocation literature [29], [38], [39]. As a result, the interference outage constraint to the PU can be neglected in the sequel, as we focus on the requirement of secure communication relying on the constraints (18b)-(18d).

Remark 2: It should be noted that a long-term interference outage probability constraint is considered in this paper to guarantee the QoS of the PU. While for the real-time applications, a short-term instantaneous interference constraint would be a more suitable choice [40], [41].

\section{Robust Beamforming Design With Outage CONSTRAINTS}

We note that the problem given by (18) is non-convex due to the outage constraints in (18b)-(18d) do not admit tractable closed-form expressions, which is very challenging and complicated to solve problem (18) directly. To overcome this predicament, we focus on how to transform the outage constraints (18b)-(18d) into equivalent yet more tractable forms. There are in general two popularly used methods for robust optimization problem with probabilistic constraints: probability distribution method and approximation method [42]. In what follows, we develop two approaches to solve problem (18) respectively by SDP relaxation, and then the tightness of the applied rank-relaxation is further investigated.

\section{A. S-Procedure Based Method}

We start by defining new matrix $\mathbf{W}=\mathbf{w w}^{H}$ with $\operatorname{rank}(\mathbf{W})=1$ and $\mathbf{W} \succeq \mathbf{0} \in \mathbb{C}^{N_{t} \times N_{t}}$. Then, the output SINR of the PU in (18b), i.e., $\operatorname{SINR}_{p} \geq \gamma_{p}$, can be expressed in terms of $\mathbf{W}$ as

$$
\frac{1}{\gamma_{p}} P_{p}\left|h_{p}\right|^{2} \geq\left(\hat{\mathbf{g}}_{p}+\Delta \mathbf{g}_{p}\right)^{H} \mathbf{W}\left(\hat{\mathbf{g}}_{p}+\Delta \mathbf{g}_{p}\right)+\sigma_{p}^{2} .
$$

Substituting (15) into (19) and rearranging the term, we can obtain

$$
\left(\hat{\mathbf{g}}_{p}+\boldsymbol{\Omega}_{p}^{1 / 2} \mathbf{r}_{p}\right)^{H} \mathbf{A}\left(\hat{\mathbf{g}}_{p}+\boldsymbol{\Omega}_{p}^{1 / 2} \mathbf{r}_{p}\right)+\frac{1}{\gamma_{p}} P_{p}\left|h_{p}\right|^{2}-\sigma_{p}^{2} \geq 0,
$$

where $\mathbf{A}=-\mathbf{W}$. To further proceed, we introduce the following lemma [43]. 
Lemma 1: For an arbitrary set $\mathcal{B} \in \mathbb{C}^{N_{t} \times 1}$ satisfying $\operatorname{Pr}\{\mathbf{a} \in$ $\mathcal{B}\} \geq 1-\rho$, we have

$$
\begin{aligned}
& \mathbf{a}^{H} \mathbf{Q a}+2 \operatorname{Re}\left(\mathbf{a}^{H} \mathbf{r}\right)+c \geq 0, \forall \mathbf{a} \in \mathcal{B}, \\
& \Longrightarrow \operatorname{Pr}\left\{\mathbf{a}^{H} \mathbf{Q} \mathbf{a}+2 \operatorname{Re}\left(\mathbf{a}^{H} \mathbf{r}\right)+c \geq 0\right\} \geq 1-\rho .
\end{aligned}
$$

Using Lemma 1, constraint (18b) is transformed into the following deterministic quadratic constraint:

$$
\begin{aligned}
f\left(\mathbf{r}_{p}\right) \triangleq \mathbf{r}_{p}^{H} \boldsymbol{\Omega}_{p}^{1 / 2} \mathbf{A} \boldsymbol{\Omega}_{p}^{1 / 2} \mathbf{r}_{p}+ & 2 \operatorname{Re}\left\{\mathbf{r}_{p}^{H} \boldsymbol{\Omega}_{p}^{1 / 2} \mathbf{A} \hat{\mathbf{g}}_{p}\right\}+\hat{\mathbf{g}}_{p}^{H} \mathbf{A} \hat{\mathbf{g}}_{p} \\
& +\frac{1}{\gamma_{p}} P_{p}\left|h_{p}\right|^{2}-\sigma_{p}^{2} \geq 0 .
\end{aligned}
$$

It is noted that $\mathbf{r}_{p}$ is located in the sphere set $\mathcal{B}_{p}=$ $\left\{\mathbf{r}_{p} \in \mathbb{C}^{N_{t} \times 1} \mid\left\|\mathbf{r}_{p}\right\|^{2} \leq R_{p}^{2}\right\}$, where $R_{p}$ is the sphere radius. According to [30], it is sufficient for $\operatorname{Pr}\left\{f\left(\mathbf{r}_{p}\right) \geq 0\right\} \geq 1-\rho_{p}$ to hold if $\operatorname{Pr}\left\{\mathbf{r}_{p} \in \mathcal{B}_{p} \geq 1-\rho_{p}\right\}$. Recall that $\mathbf{r}_{p} \sim \mathcal{C N}\left(\mathbf{0}, \mathbf{I}_{N_{t}}\right)$, $R_{p}$ can be obtained by $R_{p}=\sqrt{\frac{\mathcal{I}_{m}\left(1-\rho_{p}\right)}{2}}$, where $\mathcal{I}_{m}(\cdot)$ represents the inverse cumulative distribution function of the Chisquare random variable and the degree-of-freedom is $m=2 N_{t}$.

Similarly, applying Lemma 1, the constraints in (18c) and (18d) are respectively transformed into the following deterministic quadratic constraints:

$$
\begin{aligned}
f\left(\mathbf{r}_{e}\right) \triangleq \mathbf{r}_{e}^{H} \boldsymbol{\Omega}_{e}^{1 / 2} \mathbf{W} \boldsymbol{\Omega}_{e}^{1 / 2} \mathbf{r}_{e}+ & 2 \operatorname{Re}\left\{\mathbf{r}_{e}^{H} \boldsymbol{\Omega}_{e}^{1 / 2} \mathbf{W} \hat{\mathbf{g}}_{e}\right\}+\hat{\mathbf{g}}_{e}^{H} \mathbf{W} \hat{\mathbf{g}}_{e} \\
+ & \sigma_{e}^{2}-\frac{1}{\gamma_{e}} P_{p}\left|h_{e}\right|^{2} \geq 0 \\
f\left(\mathbf{r}_{s}\right) \triangleq \mathbf{r}_{s}^{H} \boldsymbol{\Omega}_{s}^{1 / 2} \mathbf{W} \boldsymbol{\Omega}_{s}^{1 / 2} \mathbf{r}_{s}+ & 2 \operatorname{Re}\left\{\mathbf{r}_{s}^{H} \boldsymbol{\Omega}_{s}^{1 / 2} \mathbf{W} \hat{\mathbf{g}}_{s}\right\}+\hat{\mathbf{g}}_{s}^{H} \mathbf{W} \hat{\mathbf{g}}_{s} \\
& -\gamma_{s}\left(P_{p}\left|h_{s}\right|^{2}+\sigma_{s}^{2}\right) \geq 0,
\end{aligned}
$$

where $\mathbf{r}_{e}$ and $\mathbf{r}_{s}$ are bounded to the sphere sets $\mathcal{B}_{e}=\left\{\mathbf{r}_{e} \in \mathbb{C}^{N_{t} \times 1} \mid\left\|\mathbf{r}_{e}\right\|^{2} \leq R_{e}^{2}\right\} \quad$ and $\quad \mathcal{B}_{s}=$ $\left\{\mathbf{r}_{s} \in \mathbb{C}^{N_{t} \times 1} \mid\left\|\mathbf{r}_{s}\right\|^{2} \leq R_{s}^{2}\right\}, R_{e}$ and $R_{s}$ are the corresponding sphere radiuses respectively.

In this setup, incorporating (23)-(25), the power minimization problem (18) can be equivalently recast as

$$
\begin{array}{ll} 
& \min _{\mathbf{W} \succeq \mathbf{0}} \operatorname{Tr}(\mathbf{W}) \\
\text { s.t. } & f\left(\mathbf{r}_{p}\right) \geq 0, \mathbf{r}_{p}^{H} \mathbf{I}_{N_{t}} \mathbf{r}_{p}-\frac{\mathcal{I}_{m}\left(1-\rho_{p}\right)}{2} \leq 0, \\
& f\left(\mathbf{r}_{e}\right) \geq 0, \mathbf{r}_{e}^{H} \mathbf{I}_{N_{t}} \mathbf{r}_{e}-\frac{\mathcal{I}_{m}\left(1-\rho_{e}\right)}{2} \leq 0, \\
& f\left(\mathbf{r}_{s}\right) \geq 0, \mathbf{r}_{s}^{H} \mathbf{I}_{N_{t}} \mathbf{r}_{s}-\frac{\mathcal{I}_{m}\left(1-\rho_{s}\right)}{2} \leq 0, \\
& \operatorname{rank}(\mathbf{W})=1 .
\end{array}
$$

While problem (26) is still non-convex, since (26b)-(26d) have infinite constraints due to the randomness of error vectors $\mathbf{r}_{p}, \mathbf{r}_{e}, \mathbf{r}_{s}$ and the rank-one constraint (26e) is non-convex, which is intractable. To make this problem tractable, with the help of $\mathcal{S}$-Procedure [44], we first transform the constraints (26b)-(26d) into linear matrix inequalities (LMIs).

Lemma 2: ( $\mathcal{S}$-Procedure [44]): Define the function

$$
f_{k}(\mathbf{x})=\mathbf{x}^{H} \mathbf{A}_{k} \mathbf{x}+2 \operatorname{Re}\left\{\mathbf{b}_{k}^{H} \mathbf{x}\right\}+c_{k}, k=1,2,
$$

where $\mathbf{A}_{k} \in \mathbb{C}^{n \times n}, \mathbf{b}_{k} \in \mathbb{C}^{n \times 1}, \mathbf{x} \in \mathbb{C}^{n \times 1}$, and $c_{k} \in \mathbb{R}$. Then, $f_{1}(\mathbf{x}) \leq 0 \Longrightarrow f_{2}(\mathbf{x}) \leq 0$ holds if and only if there exists a $\mu \geq 0$ such that

$$
\mu\left[\begin{array}{cc}
\mathbf{A}_{1} & \mathbf{b}_{1} \\
\mathbf{b}_{1}^{H} & c_{1}
\end{array}\right]-\left[\begin{array}{cc}
\mathbf{A}_{2} & \mathbf{b}_{2} \\
\mathbf{b}_{2}^{H} & c_{2}
\end{array}\right] \succeq \mathbf{0}
$$

supposed that there exists a vector $\hat{\mathbf{x}}$ such that $f_{1}(\hat{\mathbf{x}})<0$.

Based on Lemma 2 and then applying the SDR to omit the rank-one constraint (26e), the robust power minimization problem (26) can be reformulated as a convex SDP problem given by (29), as shown at the top of next page. The optimal solution to SDP (29) can be effectively found via existing optimization tools, e.g., SeduMi or CVX [44].

It is worth mentioning that the rank-one constraint on $\mathbf{W}$ has been relaxed in problem (29), whereas a key problem stemming from the relaxation is the rank condition of the obtained optimal solution. In general, the optimal solution of rank-relaxed problem may not be rank-one, and the result of the relaxed problem serves as a performance upper bound for the original problem. The approximation solution of the original problem can be achieved by applying randomization techniques. Fortunately, we can prove in the following that the optimal solution $\mathbf{W}^{*}$ of problem (29) is of rank-one. Accordingly, the obtained optimal solution of problem (29) is indeed the optimal solution of the original problem (18) and the optimal beamforming vector $\mathrm{w}^{*}$ can be achieved by calculating the eigenvalue decomposition. In other words, the SDR of problem (29) is tight, i.e., we can attain the global optimal solution.

Proposition 1: There exists a rank-one optimal solution, denoted as $\mathbf{W}^{*}$, to SDR of problem (29).

Proof: Please see Appendix A.

\section{B. Bernstein-Type Inequality Based Method}

Despite the method aforementioned benefits from the computational efficiency owing to the convex approximation, the $\mathcal{S}$ Procedure approach is less conservative. To find a better approximation, in this subsection, we propose to employ the Bernsteininequality approach as a tool to tackle problem (18) by deriving a series of approximate expressions to the probabilistic constraints. The comparison of $\mathcal{S}$-Procedure and Bernstein-typeinequality approaches is even more important in CSTN due to this inherent difference between the two approaches.

To start with, to circumvent this difficulty of probabilistic constraints (18b)-(18d), using the Bernstein-type inequality technique [43], the non-convex expressions can be reduced to deterministic versions. This technique is given by following the lemma.

Lemma 3: For a three-field notation $(\mathbf{Q}, \mathbf{r}, c) \in \mathbb{H}^{n} \times \mathbb{C}^{n} \times \mathbb{R}$, $\mathbf{s} \sim \mathcal{C N}\left(\mathbf{0}, \mathbf{I}_{n}\right)$ and $\eta \in(0,1]$, the sufficient condition for

$$
\operatorname{Pr}\left\{\mathbf{s}^{H} \mathbf{Q} \mathbf{s}+2 \operatorname{Re}\left(\mathbf{s}^{H} \mathbf{r}\right)+c \geq 0\right\} \geq 1-\eta
$$

is equivalent to the following series of linear matrix inequality 


$$
\begin{array}{ll} 
& \min _{\mathbf{W}, \mu_{p}, \mu_{e}, \mu_{s}} \operatorname{Tr}(\mathbf{W}) \\
\text { s.t. } & {\left[\begin{array}{cc}
\boldsymbol{\Omega}_{p}^{1 / 2} \mathbf{A} \boldsymbol{\Omega}_{p}^{1 / 2}+\mu_{p} \mathbf{I}_{N_{t}} & \boldsymbol{\Omega}_{p}^{1 / 2} \mathbf{A} \hat{\mathbf{g}}_{p} \\
\hat{\mathbf{g}}_{p}^{H} \mathbf{A} \boldsymbol{\Omega}_{p}^{1 / 2} & \hat{\mathbf{g}}_{p}^{H} \mathbf{A} \hat{\mathbf{g}}_{p}+\frac{P_{p}}{\gamma_{p}}\left|h_{p}\right|^{2}-\sigma_{p}^{2}-\mu_{p} \frac{\mathcal{I}_{m}\left(1-\rho_{p}\right)}{2}
\end{array}\right] \geq \mathbf{0},} \\
& {\left[\begin{array}{cc}
\boldsymbol{\Omega}_{e}^{1 / 2} \mathbf{W} \boldsymbol{\Omega}_{e}^{1 / 2}+\mu_{e} \mathbf{I}_{N_{t}} & \boldsymbol{\Omega}_{e}^{1 / 2} \mathbf{W} \hat{\mathbf{g}}_{e} \\
\hat{\mathbf{g}}_{e}^{H} \mathbf{W} \boldsymbol{\Omega}_{e}^{1 / 2} & \hat{\mathbf{g}}_{e}^{H} \mathbf{W} \hat{\mathbf{g}}_{e}+\sigma_{e}^{2}-\frac{P_{p}}{\gamma_{e}}\left|h_{e}\right|^{2}-\mu_{e} \frac{\mathcal{I}_{m}\left(1-\rho_{e}\right)}{2}
\end{array}\right] \geq \mathbf{0},} \\
& {\left[\begin{array}{cc}
\boldsymbol{\Omega}_{s}^{1 / 2} \mathbf{W} \boldsymbol{\Omega}_{s}^{1 / 2}+\mu_{s} \mathbf{I}_{N_{t}} & \boldsymbol{\Omega}_{s}^{1 / 2} \mathbf{W} \hat{\mathbf{g}}_{s} \\
\hat{\mathbf{g}}_{s}^{H} \mathbf{W} \boldsymbol{\Omega}_{s}^{1 / 2} & \hat{\mathbf{g}}_{s}^{H} \mathbf{W} \hat{\mathbf{g}}_{s}-\gamma_{s}\left(P_{p}\left|h_{s}\right|^{2}+\sigma_{s}^{2}\right)-\mu_{s} \frac{\mathcal{I}_{m}\left(1-\rho_{s}\right)}{2}
\end{array}\right] \geq \mathbf{0},} \\
& \mathbf{W} \succeq \mathbf{0}, \mu_{p} \geq 0, \mu_{e} \geq 0, \mu_{s} \geq 0 .
\end{array}
$$

(LMI) and second-order cone (SOC) constraints:

$$
\left\{\begin{array}{l}
\operatorname{tr}(\mathbf{Q})-\sqrt{-2 \ln \eta} t_{1}+t_{2} \ln \eta+c \geq 0, \\
\left\|\left[\begin{array}{c}
\operatorname{vec}(\mathbf{Q}) \\
\sqrt{2} \mathbf{r}
\end{array}\right]\right\| \leq t_{1}, \\
t_{2} \mathbf{I}_{n}+\mathbf{Q} \succeq \mathbf{0}, t_{2} \geq 0,
\end{array}\right.
$$

where $t_{1}$ and $t_{2}$ are the slack variables.

By applying the results of Lemma 3 and introducing the slack variables $\lambda_{p} \geq 0$ and $\eta_{p} \geq 0$, the SINR outage constraint of the PU, i.e., $\operatorname{Pr}\left\{f\left(\mathbf{r}_{p}\right) \geq \gamma_{p}\right\} \geq 1-\rho_{p}$, can be equivalently converted into a sequence of finite convex constraints as

$$
\left\{\begin{array}{l}
\operatorname{Tr}\left(\boldsymbol{\Omega}_{p}^{1 / 2} \mathbf{A} \boldsymbol{\Omega}_{p}^{1 / 2}\right)-\sqrt{-2 \ln \rho_{p}} \lambda_{p}+\eta_{p} \ln \rho_{p}+c_{p} \geq 0, \\
\left\|\left[\begin{array}{c}
\operatorname{vec}\left(\boldsymbol{\Omega}_{p}^{1 / 2} \mathbf{A} \boldsymbol{\Omega}_{p}^{1 / 2}\right) \\
\sqrt{2} \boldsymbol{\Omega}_{p}^{1 / 2} \mathbf{A} \hat{\mathbf{g}}_{p}
\end{array}\right]\right\| \leq \lambda_{p}, \\
\eta_{p} \mathbf{I}_{N_{t}}+\boldsymbol{\Omega}_{p}^{1 / 2} \mathbf{A} \boldsymbol{\Omega}_{p}^{1 / 2} \succeq \mathbf{0}, \eta_{p} \geq 0,
\end{array}\right.
$$

where $c_{p}=\hat{\mathbf{g}}_{p}^{H} \mathbf{A} \hat{\mathbf{g}}_{p}+\frac{P_{p}}{\gamma_{p}}\left|h_{p}\right|^{2}-\sigma_{p}^{2}$.

Using similar step for the tolerant leakage-SINR outage constraint of the Eve, i.e., $\operatorname{Pr}\left\{f\left(\mathbf{r}_{e}\right) \leq \gamma_{e}\right\} \geq 1-\rho_{e}$, it can also be equivalently converted into a sequence of finite convex constraints as

$$
\left\{\begin{array}{l}
\operatorname{Tr}\left(\boldsymbol{\Omega}_{e}^{1 / 2} \mathbf{W} \boldsymbol{\Omega}_{e}^{1 / 2}\right)-\sqrt{-2 \ln \rho_{e}} \lambda_{e}+\eta_{e} \ln \rho_{e}+c_{e} \geq 0, \\
\left\|\left[\begin{array}{c}
\operatorname{vec}\left(\boldsymbol{\Omega}_{e}^{1 / 2} \mathbf{W} \boldsymbol{\Omega}_{e}^{1 / 2}\right) \\
\sqrt{2} \boldsymbol{\Omega}_{e}^{1 / 2} \mathbf{W} \hat{\mathbf{g}}_{e}
\end{array}\right]\right\| \leq \lambda_{e}, \\
\eta_{e} \mathbf{I}_{N_{t}}+\boldsymbol{\Omega}_{e}^{1 / 2} \mathbf{W} \boldsymbol{\Omega}_{e}^{1 / 2} \succeq \mathbf{0}, \eta_{e} \geq 0,
\end{array}\right.
$$

where $c_{e}=\hat{\mathbf{g}}_{e}^{H} \mathbf{W} \hat{\mathbf{g}}_{e}+\sigma_{e}^{2}-\frac{P_{p}}{\gamma_{e}}\left|h_{e}\right|^{2} \cdot \lambda_{e} \geq 0$ and $\eta_{e} \geq 0$ are the introduced the slack variables.

As for the SINR outage constraint of the SU, i.e., $\operatorname{Pr}\left\{f\left(\mathbf{r}_{s}\right) \geq \gamma_{s}\right\} \geq 1-\rho_{s}$, by introducing slack variables $\lambda_{s} \geq 0, \eta_{s} \geq 0$ and applying the results of Lemma 3 , it can be recast as

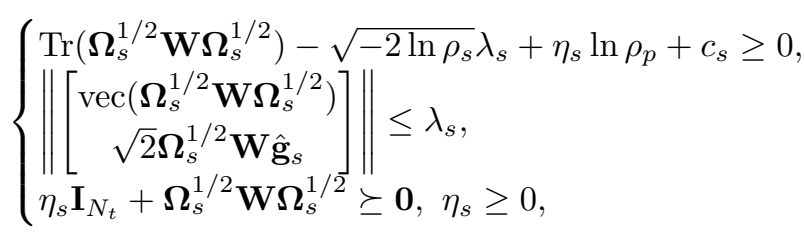

where $c_{s}=\hat{\mathbf{g}}_{s}^{H} \mathbf{W} \hat{\mathbf{g}}_{s}-\gamma_{s}\left(P_{p}\left|h_{s}\right|^{2}+\sigma_{s}^{2}\right)$.
Incorporating (32)-(34) and applying the rank-one relaxation, the corresponding robust optimization problem is reformulated as the following convex SDP, i.e.,

$$
\begin{array}{ll} 
& \min \operatorname{Wr}(\mathbf{W}) \\
\text { s.t. } & (32),(33) \text { and }(34), \\
& \mathbf{W} \succeq \mathbf{0}, \lambda_{p} \geq 0, \eta_{p} \geq 0, \\
& \lambda_{e} \geq 0, \eta_{e} \geq 0, \lambda_{s} \geq 0, \eta_{s} \geq 0 .
\end{array}
$$

Remarkably, the optimal solution to the convex problem (35) can be found by using the convex optimization solvers, e.g., SeduMi or CVX [44]. Since the rank-one constraint on W has been relaxed in problem (35), the attained optimal solution may not be rank one. In the following, we can prove that the optimal solution $\mathbf{W}^{*}$ of (35) satisfies the condition of rank-one. Accordingly, the obtained optimal solution to the rank relaxation of problem (35) is indeed the optimal solution to the original problem (18). In other words, the rank relaxation of problem (35) is tight, i.e., we can achieve the global optimal solution.

Proposition 2: There exists a rank-one optimal solution, denoted as $\mathbf{W}^{*}$, to SDR of problem (35).

The proof is similar to the Appendix B in [30], we omit it here for brevity.

\section{Computational Complexity}

In this part, the computational complexities of the proposed robust secure schemes are analyzed. It is worth mentioning that the convex restriction formulations (29) and (35) involve only LMI and SOC constraints, and hence we apply standard interiorpoint methods to solve them. According to [43], the complexity is composed of two parts, namely, iteration complexity and the per-iteration computation cost. Specially, the computational complexity per-iteration mainly arises from the number of optimization variables, the number of LMI constraints and the size, the number of the SOC constraints and the size. For the $\mathcal{S}$ Procedure based method, the optimization problem (29) has $N_{t}^{2}$ design variables and 3 slack variables, 3 LMI constraints of size $N_{t}+1$, one LMI constraint of size $N_{t}$, and 3 LMI constraints of size 1 . For the Bernstein-type-inequality based method, the optimization problem (35) has $N_{t}^{2}$ design variables and 6 slack 


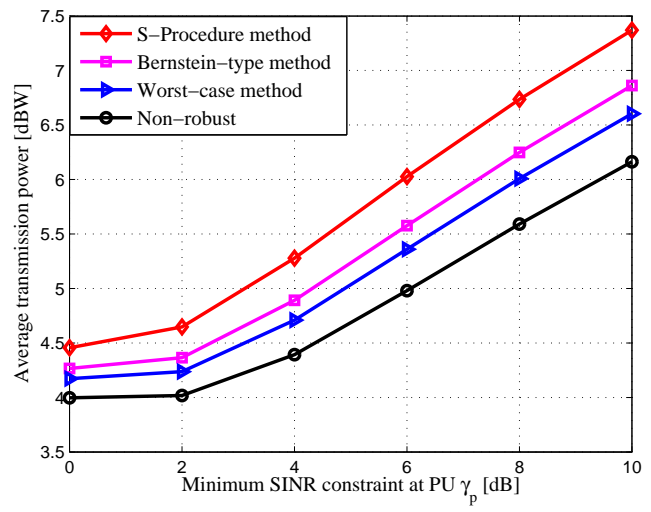

Fig. 2. Average transmission power versus minimum SINR constraint at PU $\gamma_{p}$ with $\rho=0.1$.

variables, 4 LMI constraints of size $N_{t}, 12 \mathrm{LMI}$ constraints of size 1 , and 3 SOC constraints of dimension $N_{t}^{2}+N_{t}+1$.

From Table I, we can see that the proposed methods are computationally efficient for the non-convex beamforming optimization. The computational complexity of the Bernsteintype-inequality based method is larger than that of the $\mathcal{S}$ Procedure based method, since the proposed Bernstein-typeinequality based method has a more complicated set involving the LMI and SOC constraints. However, the performance of the Bernstein-type-inequality based method is superior to that of the $\mathcal{S}$-Procedure based method in terms of power consumption, which is exhibited by numerical results presented in Section V.

\section{NUMERICAL RESUlts}

In this section, we provide simulation results for evaluating the performance of the proposed robust design methods in a CSTN scenario. We consider the scenario that the number of antennas at the terrestrial BS is set to $N_{t}=4$ unless otherwise specified, and the other communication nodes have a single antenna. It is assumed that the satellite links follow the ShadowedRician fading distribution and undergo heavy shadowing with parameters $\left(b_{i}, m_{i}, \Omega_{i}\right)=\left(0.063,2,8.97 \times 10^{-4}\right), \forall i \in\{p, e, s\}$ [45]. In our simulation setup, the beam angles between SAT and $\mathrm{PU}$, Eve, as well as $\mathrm{SU}$ are respectively set as $0.01^{\circ}, 0.4^{\circ}$, and $0.8^{\circ}$. The transmit power at SAT is set to be $P_{p}=40 \mathrm{~W}$. For the sake of simplicity, we assume that the covariance matrices of the channel error vectors are identical as $\Omega_{p}=\Omega_{e}=\Omega_{s}=\epsilon \mathbf{I}_{N_{t}}$ where $\epsilon=0.005$ is the channel error variance, and the outage probabilities are $\rho_{p}=\rho_{e}=\rho_{s}=\rho$. We set the 3-dB angle as $\varphi_{3 \mathrm{~dB}}=0.4^{\circ}$, the carrier frequency as $\lambda=2 \mathrm{GHz}$, the maximal antenna gain of SAT $b_{\max }=52 \mathrm{dBi}$, the inter-element spacing as $d=\frac{\lambda}{2}$, the AOD from terrestrial BS to $\mathrm{SU}$ as $\theta_{s}=0^{\circ}$, the AOD from terrestrial BS to PU as $\theta_{p}=40^{\circ}$, the angle spread as $\Delta \theta_{s}=\Delta \theta_{e}=5^{\circ}$ [3]. All simulation results are derived by averaging over 1000 randomly generated channel realizations.

Fig. 2 shows the average transmission power performance of the proposed $\mathcal{S}$-Procedure method, the proposed Bernsteintype method, the deterministic uncertainty model based scheme (denoted as "Worst-case" method) [46] and the non-robust

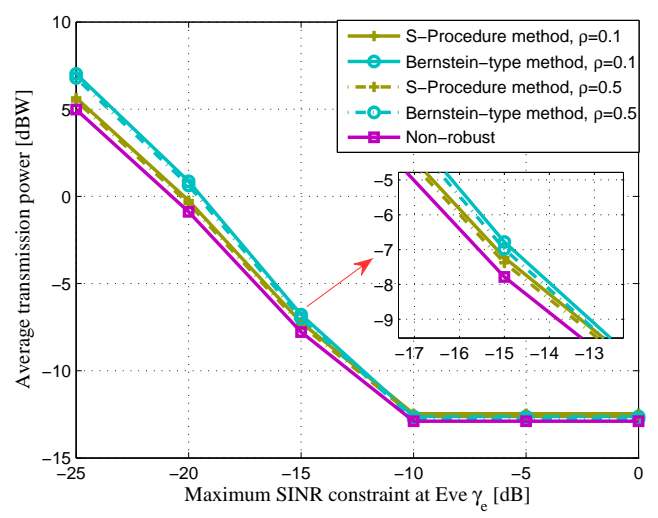

Fig. 3. Average transmission power versus maximum tolerant SINR constraint at Eve $\gamma_{e}$ with $\gamma_{p}=10 \mathrm{~dB}$ and $\gamma_{e}=0 \mathrm{~dB}$.

scheme (denoted as "Non-robust"). Note that the non-robust scheme is obtained using the nominal vectors $\hat{\mathrm{g}}_{p}, \hat{\mathrm{g}}_{e}$ and $\hat{\mathrm{g}}_{s}$. In this setup, we set the maximum tolerable SINR at Eve as $\gamma_{e}=0 \mathrm{~dB}$ and the minimum SINR requirement at $\mathrm{SU}$ as $\gamma_{s}=15 \mathrm{~dB}$. As expected, we observe from Fig. 2 that the proposed Bernstein-type method is distinctly better than the $\mathcal{S}$ Procedure method, the approximation exploited in the Bernsteintype method is tighter than that in the $\mathcal{S}$-Procedure method and the performance gain becomes more evident with increasing $\gamma_{p}$. It can also be seen that the average transmission power of the terrestrial BS increases with the minimum SINR constraint at PU, no matter whether the models of the CSI errors. This is because a higher transmission power is allocated in order to satisfy the increasing SINR requirement at PU. Compared with the non-robust design, the proposed schemes achieve robustness at the cost of slightly increased transmission power. Although the deterministic-constraint based worst-case scheme consumes less transmission power than the proposed outage constrained robust methods, the extreme scenario may happen rarely and may not reflect practical channels accurately.

Fig. 3 depicts the average transmission power against the maximum tolerant SINR requirement $\gamma_{e}$ at Eve under different $\rho$, in which the minimum SINR requirement at PU and $\mathrm{SU}$ is $\gamma_{p}=10 \mathrm{~dB}$ and $\gamma_{s}=0 \mathrm{~dB}$ respectively. It is observed that, with the increasing of $\gamma_{e}$, the average transmission power decreases and the curves tends to become stable when $\gamma_{e}$ is large. This is due to the fact that when $\gamma_{e}$ is large enough, the SINR constraint at Eve becomes independent of the beamforming design and in such a case the network will be reduced to that without secrecy. From Fig. 3, we can also see that the performance loss between the proposed robust design methods and the non-robust design method is small. Furthermore, it is interesting to mention that the robust secure beamforming designs have a slight impact on large outage probability $\rho$.

Next, we compare the power consumption performance of the proposed robust design methods for different number of transmit antennas associated with $N_{t}=4$ and $N_{t}=6$, respectively. The parameter settings in this setup are the same as those in Fig. 2. As shown in Fig. 4, it is obvious that the transmission 
TABLE I

COMPUTATIONAL COMPLEXITY ANALYSIS.

\begin{tabular}{|l|l|}
\hline Methods & Computation Complexity Order (Ignoring $\ln (1 / \epsilon)$ in $\mathcal{O}(\cdot) \ln (1 / \epsilon)$, where $\epsilon$ denotes an accuracy). \\
\hline $\mathcal{S}$-Procedure & $\mathcal{O}\left(\sqrt{4 N_{t}+6} \cdot n \cdot\left[3\left(N_{t}+1\right)^{2}\left(N_{t}+1+n\right)+N_{t}^{2}\left(N_{t}+n\right)+3+n+n^{2}\right]\right)$, where $n=\mathcal{O}\left(N_{t}^{2}+3\right)$. \\
\hline Bernstein-type-inequality & $\mathcal{O}\left(\sqrt{4 N_{t}+15} \cdot n \cdot\left[4 N_{t}^{2}\left(N_{t}+n\right)+3\left(N_{t}^{2}+N_{t}+2\right)+12+12 n+n^{2}\right]\right)$, where $n=\mathcal{O}\left(N_{t}^{2}+6\right)$. \\
\hline
\end{tabular}

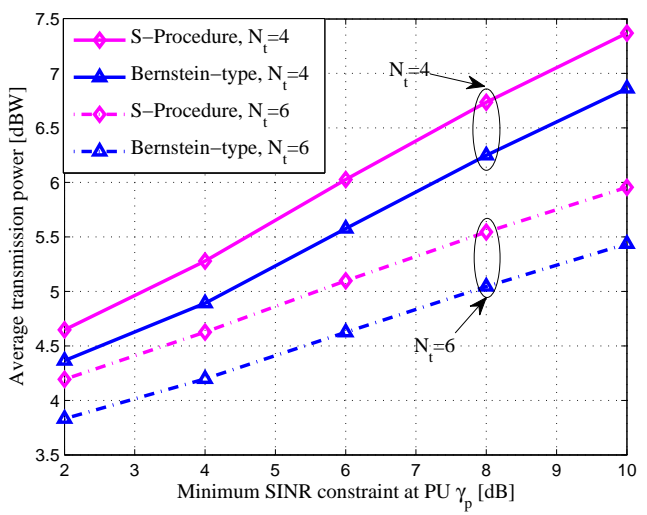

Fig. 4. Average transmission power versus minimum SINR constraint at PU $\gamma_{p}$ for $N_{t}=4$ and $N_{t}=6$, respectively.

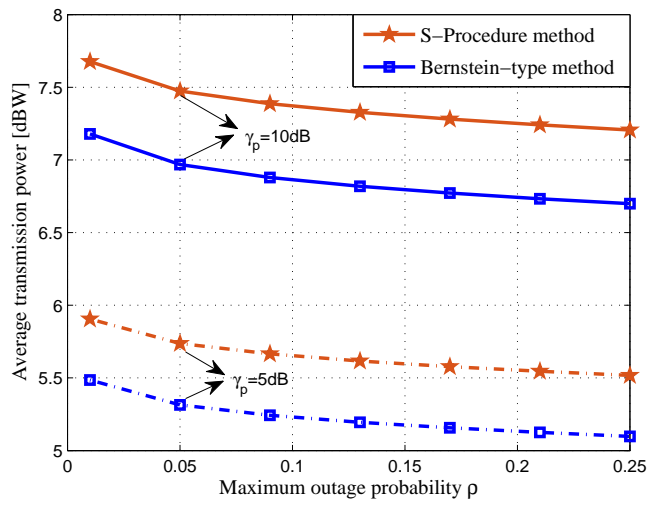

Fig. 5. Average transmission power versus maximum outage probability $\rho$.

power of the proposed design methods greatly degrades with the increasing of the transmit antennas $N_{t}$ at the terrestrial BS. The result indicates the benefit of applying more transmit antennas for beamforming design in the MISO system. When the value of $\gamma_{p}$ increases, the performance gaps become large gradually, which implies that the proposed robust design methods are very sensitive to the SINR constraint at PU. In the high SINR region, we can clearly see that the proposed Bernstein-type method with $N_{t}=6$ can save $1.4 \mathrm{dBW}$ power compared with this method with $N_{t}=4$ due to the optimized transmission. Similarly, almost the same power consumption can be saved in the proposed $\mathcal{S}$-Procedure method.

We further probe into the relationship between the transmission power and the maximum outage probability $\rho$ in Fig. 5 when $\rho$ ranges from 0.01 to 0.25 and the minimum SINR

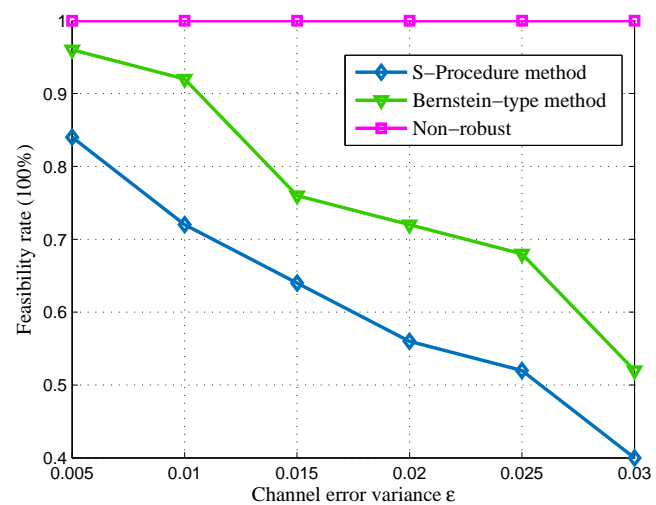

Fig. 6. Feasibility rate versus channel error variance $\epsilon$.

constraint at PU $\gamma_{p}$ are respectively set as $5 \mathrm{~dB}$ and $10 \mathrm{~dB}$. As we can see in the figure, the average transmission power of the proposed methods degrades with the increasing of maximum outage probability $\rho$ for different $\gamma_{p}$, and the performance gap of the two proposed methods stays parallel in the whole outage probability region. For fixed outage probability $\rho$, the observation we have is that the average transmission power under $\gamma_{p}=10 \mathrm{~dB}$ is superior to that under $\gamma_{p}=5 \mathrm{~dB}$, This is reasonable, because more transmission power is required for meeting the SINR constraint at PU.

To further assess the effectiveness of our robust transmit design, we show the feasible rate comparison versus channel error variance $\epsilon$ where the results are averaged over 1000 trials and we set $\gamma_{p}=10 \mathrm{~dB}, \gamma_{e}=0 \mathrm{~dB}, \gamma_{s}=15 \mathrm{~dB}$ and $\rho=0.1$ for the robust design methods. As the results shown in Fig. 6, one can see that the feasibility rate of the $\mathcal{S}$-Procedure method is lower compared to the Bernstein-type method, which is due to the use of less variables in the $\mathcal{S}$-Procedure method. While the feasible rate of non-robust method is always one since the channel errors do not exist when assuming perfect CSI. In addition, it can be found that with the increasing of channel channel error variance $\epsilon$, the feasibility rates of the proposed methods significantly decrease. The reason behind this phenomenon lies in the fact that the robust schemes are very sensitive to the channel error variance $\epsilon$.

\section{CONCLUSION}

In this paper, the secure robust beamforming design in a CST$\mathrm{N}$ was investigated with the consideration of channel uncertainty. Based on the probabilistic CSI error model, we presented an outage-constrained robust formulation where the channel errors 
were Gaussian distributed, and the objective was to minimize the transmit power while satisfying the required constraints. Due to the presence of the probabilistic constraints, the original power minimization problem did not seem tractable directly. To address the non-convexity of the formulated optimization problem, we transformed the highly intractable constraints into a series of convex forms via advanced matrix inequality techniques, namely $\mathcal{S}$-Procedure and Bernstein-type inequality restriction techniques, resulting in safe approximate solutions. We further showed that the computational complexity of the $\mathcal{S}$-Procedure method was inferior to that of the Bernstein-type inequality method. Finally, numerical results have demonstrated the validity of the proposed schemes.

\section{APPENDIX A \\ Proof OF THEOREM 1}

To prove the rank condition of $\mathbf{W}^{*}$, we first rewrite the robust optimization problem (29) as

$$
\begin{array}{ll} 
& \min _{\mathbf{W}, \mu_{p}, \mu_{e}, \mu_{s}} \operatorname{Tr}(\mathbf{W}) \\
\text { s.t. } & \mathbf{D}\left(\mu_{p}\right)+\boldsymbol{\Gamma}_{p}^{H} \mathbf{A} \boldsymbol{\Gamma}_{p} \succeq \mathbf{0}, \\
& \mathbf{D}\left(\mu_{e}\right)+\boldsymbol{\Gamma}_{e}^{H} \mathbf{W} \boldsymbol{\Gamma}_{e} \succeq \mathbf{0}, \\
& \mathbf{D}\left(\mu_{s}\right)+\boldsymbol{\Gamma}_{s}^{H} \mathbf{W} \boldsymbol{\Gamma}_{s} \succeq \mathbf{0}, \\
& \mathbf{W} \succeq \mathbf{0}, \mu_{p} \geq 0, \mu_{e} \geq 0, \mu_{s} \geq 0,
\end{array}
$$

where

$$
\begin{aligned}
& \mathbf{D}\left(\mu_{p}\right)=\left[\begin{array}{cc}
\mu_{p} \mathbf{I}_{N_{t}} & \mathbf{0}_{N_{t} \times 1} \\
\mathbf{0}_{1 \times N_{t}} & \frac{P_{p}}{\gamma_{p}}\left|h_{p}\right|^{2}-\sigma_{p}^{2}-\mu_{p} \frac{\mathcal{I}_{m}\left(1-\rho_{p}\right)}{2}
\end{array}\right] \\
& \mathbf{D}\left(\mu_{e}\right)=\left[\begin{array}{lc}
\mu_{e} \mathbf{I}_{N_{t}} & \mathbf{0}_{N_{t} \times 1} \\
\mathbf{0}_{1 \times N_{t}} & \sigma_{e}^{2}-\frac{P_{p}}{\gamma_{e}}\left|h_{e}\right|^{2}-\mu_{e} \frac{\mathcal{I}_{m}\left(1-\rho_{e}\right)}{2}
\end{array}\right] \\
& \mathbf{D}\left(\mu_{s}\right)=\left[\begin{array}{lc}
\mu_{s} \mathbf{I}_{N_{t}} & \mathbf{0}_{N_{t} \times 1} \\
\mathbf{0}_{1 \times N_{t}} & -P_{p}\left|h_{s}\right|^{2}-\sigma_{s}^{2}-\mu_{s} \frac{\mathcal{I}_{m}\left(1-\rho_{s}\right)}{2}
\end{array}\right] \\
& \boldsymbol{\Gamma}_{p}=\left[\boldsymbol{\Omega}_{p}^{1 / 2}, \hat{\mathbf{g}}_{p}\right], \boldsymbol{\Gamma}_{e}=\left[\boldsymbol{\Omega}_{e}^{1 / 2}, \hat{\mathbf{g}}_{e}\right], \boldsymbol{\Gamma}_{s}=\left[\boldsymbol{\Omega}_{s}^{1 / 2}, \hat{\mathbf{g}}_{s}\right]
\end{aligned}
$$

Let $\boldsymbol{\Xi}=\left\{\mathbf{Z}_{p}, \mathbf{Z}_{e}, \mathbf{Z}_{s}, \mathbf{T}\right\}$ denote the collection of dual variables, where $\mathbf{Z}_{p} \succeq \mathbf{0}, \mathbf{Z}_{e} \succeq \mathbf{0}, \mathbf{Z}_{s} \succeq \mathbf{0}$ and $\mathbf{T} \succeq \mathbf{0}$ are corresponding to the constraints (36b)-(36e), the Lagrangian of problem (36) with respect to $\mathbf{W}$ can be derived by

$$
\begin{aligned}
\mathcal{L}(\boldsymbol{\Xi}, \mathbf{W})=\operatorname{Tr}(\mathbf{W}) & -\operatorname{Tr}\left[\mathbf{Z}_{p}\left(\mathbf{D}\left(\mu_{p}\right)+\boldsymbol{\Gamma}_{p}^{H} \mathbf{Z}_{p} \boldsymbol{\Gamma}_{p}\right)\right] \\
& -\operatorname{Tr}\left[\mathbf{Z}_{e}\left(\mathbf{D}\left(\mu_{e}\right)+\boldsymbol{\Gamma}_{e}^{H} \mathbf{Z}_{e} \boldsymbol{\Gamma}_{e}\right)\right] \\
& -\operatorname{Tr}\left[\mathbf{Z}_{s}\left(\mathbf{D}\left(\mu_{s}\right)+\boldsymbol{\Gamma}_{s}^{H} \mathbf{Z}_{s} \boldsymbol{\Gamma}_{s}\right)\right] \\
& -\operatorname{Tr}[\mathbf{T W}] .
\end{aligned}
$$

With the Lagrangian dual function, the dual problem of (36) is presented by

$$
\max _{\mathbf{Z}_{p} \succeq \mathbf{0}, \mathbf{Z}_{e} \succeq \mathbf{0}, \mathbf{Z}_{s} \succeq \mathbf{0}, \mathbf{T} \succeq \mathbf{0}} \min _{\mathbf{W} \succeq \mathbf{0}} \mathcal{L}(\boldsymbol{\Xi}, \mathbf{W}) .
$$

It is noted that the primal problem (36) is convex with a strict feasible point and it satisfies the Slater's condition, which implies that the strong duality holds between problem (36) and its dual, i.e., the duality gap tends to zero. This guarantees that the optimal solution to problem (36) can be attained by solving (42).

Denote the optimal solution of problem (42) by $\boldsymbol{\Xi}^{*}=$ $\left\{\mathbf{Z}_{p}^{*}, \mathbf{Z}_{e}^{*}, \mathbf{Z}_{s}^{*}, \mathbf{T}^{*}\right\}$, then the optimal solution to problem (36), $\mathbf{W}^{*}$, can be found by solving the following form:

$$
\min _{\mathbf{W} \succeq \mathbf{0}} \mathcal{L}\left(\boldsymbol{\Xi}^{*}, \mathbf{W}\right) \text {. }
$$

For ease of exposition, $\mathcal{L}\left(\boldsymbol{\Xi}^{*}, \mathbf{W}\right)$ can be expressed, after some mathematical manipulations, as

$$
\mathcal{L}\left(\boldsymbol{\Xi}^{*}, \mathbf{W}\right)=\operatorname{Tr}(\boldsymbol{\Lambda} \mathbf{W})+\ell,
$$

where

$$
\begin{gathered}
\boldsymbol{\Lambda}=\mathbf{I}_{N_{t}}+\boldsymbol{\Gamma}_{p} \mathbf{Z}_{p}^{*} \boldsymbol{\Gamma}_{p}^{H}-\boldsymbol{\Gamma}_{e} \mathbf{Z}_{e}^{*} \boldsymbol{\Gamma}_{e}^{H}-\frac{1}{\gamma_{s}} \boldsymbol{\Gamma}_{s} \mathbf{Z}_{s}^{*} \boldsymbol{\Gamma}_{s}^{H}-\mathbf{T}^{*}, \\
\ell=-\operatorname{Tr}\left[\mathbf{Z}_{p} \mathbf{D}\left(\mu_{p}\right)\right]-\operatorname{Tr}\left[\mathbf{Z}_{e} \mathbf{D}\left(\mu_{e}\right)\right]-\operatorname{Tr}\left[\mathbf{Z}_{s} \mathbf{D}\left(\mu_{s}\right)\right] .
\end{gathered}
$$

Since problem (29) is feasible, the optimal level of the equivalence problem (36) is not less than zero. Moreover, the optimal duality gap between primary problem (36) and its Lagrange dual problem (42) is zero. As a result, $\boldsymbol{\Lambda}$ is certainly positive semi-definite, i.e., $\boldsymbol{\Lambda} \succeq \mathbf{0}$, to guarantee that the Lagrangian dual function has a lower bound, i.e., the Lagrangian dual function cannot tend to $-\infty$. To proceed further, the following theorem is introduced:

Theorem 1: Given Hermitian matrix $\mathbf{W} \in \mathbb{C}^{N_{t} \times N_{t}}$, if $\operatorname{rank}(\mathbf{W})=K \leq N_{t}$, then $\mathbf{W}$ can be derived as $\mathbf{W}=$ $\sum_{k=1}^{K} v_{k} \mathbf{a}_{k} \mathbf{a}_{k}^{H}$, where $v_{k}$ and $\mathbf{a}_{k}$ are the $k$-th non-zero eigenvalue and the $k$-th eigenvector related to $\mathbf{W}$, respectively.

Following the similar procedure of [30], the rank-one property of the optimal solution to (44) can be explicitly verified.

\section{REFERENCES}

[1] Y. Kawamoto, Z. M. Fadlullah, H. Nishiyama, N. Kato, and M. Toyoshima, "Prospects and challenges of context-aware multimedia content delivery in cooperative satellite and terrestrial networks," IEEE Commun. Mag., vol. 52, no. 6, pp. 55-61, Feb. 2014.

[2] C. Niephaus, M. Kretschmer, and G. Ghinea, "QoS provisioning in converged satellite and terrestrial networks: A survey of the state-of-theart," IEEE Commun. Surveys Tuts., vol. 18, no. 4, pp. 2415-2441, Fourth Quarter 2016.

[3] K. An, M. Lin, J. Ouyang, and W.-P. Zhu, "Secure transmission in cognitive satellite terrestrial networks," IEEE J. Sel. Areas Commun., vol. 34, no. 11, pp. 3025-3037, Nov. 2016.

[4] S. Vassaki, M. I. Poulakis, A. D. Panagopoulos, and P. Constantinou, "Power allocation in cognitive satellite terrestrial networks with QoS constraints," IEEE Commun. Lett., vol. 17, no. 7, pp. 1344-1347, Jul. 2013.

[5] A. Roy-Chowdhury, J. S. Baras, M. Hadjitheodosiou, and S. Papademetriou, "Security issues in hybrid networks with a satellite component," IEEE Wireless Commun., vol. 12, no. 6, pp. 50-61, Dec. 2005.

[6] Z. Chu, H. Xing, M. Johnston, and S. Y. L. Goff, "Secrecy rate optimizations for a MISO secrecy channel with multiple multiantenna eavesdroppers," IEEE Trans. Wireless Commun., vol. 15, no. 1, pp. 283 297, Jan. 2016.

[7] Z. Chu, K. Cumanan, Z. Ding, M. Johnston, and S. Y. L. Goff, "Secrecy rate optimizations for a MIMO secrecy channel with a cooperative jammer," IEEE Trans. Veh. Technol., vol. 64, no. 5, pp. 1833-1847, May 2015. 
[8] B. Li, Z. Fei, and H. Chen, "Robust artificial noise-aided secure beamforming in wireless-powered non-regenerative relay," IEEE Access, vol. 4, pp. 7921-7929, Nov. 2016.

[9] A. Kalantari, S. Maleki, G. Zheng, S. Chatzinotas, and B. Ottersten, "Joint power control in wiretap interference channels," IEEE Trans. Wireless Commun., vol. 14, no. 7, pp. 3810-3823, Jul. 2015.

[10] S. Maleki, A. Kalantari, S. Chatzinotas, and B. Ottersten, "Power allocation for energy-constrained cognitive radios in the presence of an eavesdropper," in Proc. IEEE International Conference on Acoustics, Speech and Signal Processing (ICASSP), Florence, Italy, May 2014, pp. 5695-5699.

[11] A. Kalantari, M. Soltanalian, S. Maleki, S. Chatzinotas, and B. Ottersten, "Directional modulation via symbol-level precoding: A way to enhance security," IEEE J. Sel. Topics Signal Process., vol. 10, no. 8, pp. 14781493, Dec. 2016.

[12] B. Evans, M. Werner, E. Lutz, M. Bousquet, G. Corazza, G. Maral, and R. Rumeau, "Integration of satellite and terrestrial systems in future multimedia communications," IEEE Wireless Commun., vol. 12, no. 5, pp. 72-80, Oct. 2005

[13] L. Yang and M. O. Hasna, "Performance analysis of amplify-and-forward hybrid satellite-terrestrial networks with cochannel interference," IEEE Trans. Commun., vol. 63, no. 12, pp. 5052-5061, Dec. 2015.

[14] M. K. Arti, "Channel estimation and detection in hybrid satellite-terrestrial communication systems," IEEE Trans. Veh. Technol., vol. 65, no. 7, pp 5764-5771, Jul. 2016.

[15] Y. Ruan, Y. Li, C.-X. Wang, R. Zhang, and H. Zhang, "Outage performance of integrated satellite-terrestrial networks with hybrid CCI," IEEE Commun. Lett., vol. 21, no. 7, pp. 1545-1548, Jul. 2017.

[16] K. An, M. Lin, J. Ouyang, Y. Huang, and G. Zheng, "Symbol error analysis of hybrid satellite-terrestrial cooperative networks with co-channel interference," IEEE Commun. Lett., vol. 18, no. 11, pp. 1947-195, Nov. 2014.

[17] K. An, M. Lin, T. Liang, J. Wang, J. Wang, Y. Huang, and A. L. Swindlehurst, "Performance analysis of multi-antenna hybrid satelliteterrestrial relay networks in the presence of interference," IEEE Trans. Commun., vol. 63, no. 11, pp. 4390-4404, Nov. 2015

[18] H. Li, H. Yin, F. Dong, X. Gong, C. Deng, L. Jia, and B. Ren, "Capacity upper bound analysis of the hybrid satellite terrestrial communication systems," IEEE Commun. Lett., vol. 20, no. 12, pp. 2402-2405, Dec. 2016.

[19] M. Jia, X. Gu, Q. Guo, W. Xiang, and N. Zhang, "Broadband hybrid satellite-terrestrial communication systems based on cognitive radio toward 5G," IEEE Wireless Commun., vol. 23, no. 6, pp. 96-106, Dec 2016.

[20] S. K. Sharma, S. Chatzinotas, and B. Ottersten, "Cognitive radio techniques for satellite communication systems," in Proc. IEEE 78th Vehicular Technology Conference (VTC Fall), Las Vegas, USA, Sep. 2013, pp. 1-5.

[21] E. Lagunas, S. K. Sharma, S. Maleki, S. Chatzinotas, and B. Ottersten, "Resource allocation for cognitive satellite communications with incumbent terrestrial networks," IEEE Trans. Cognit. Commun. Netw., vol. 1, no. 3, pp. 305-317, Sep. 2015.

[22] S. Maleki et al., "Cognitive spectrum utilization in Ka band multibeam satellite communications," IEEE Commun. Mag., vol. 53, no. 3, pp. 24-29, Mar. 2015

[23] S. Shi, G. Li, K. An, Z. Li, and G. Zheng, "Optimal power control for real-time applications in cognitive satellite terrestrial networks," IEEE Commun. Lett., vol. 21, no. 8, pp. 1815-1818, Aug. 2017.

[24] K. An, M. Lin, W.-P. Zhu, Y. Huang, and G. Zheng, "Outage performance of cognitive hybrid satellite-terrestrial networks with interference constraint," IEEE Trans. Veh. Technol., vol. 65, no. 11, pp. 9397-9404, Nov. 2016.

[25] Y. Liu, H.-H. Chen, and L. Wang, "Physical layer security for next generation wireless networks: Theories, technologies, and challenges," IEEE Commun. Surv. Tut., vol. 19, no. 1, pp. 347-376, First Quarter 2017.

[26] J. Lei, Z. Han, M. A. Vazquez-Castro, and A. Hjorungnes, "Secure satellite communication systems design with individual secrecy rate constraints," IEEE Trans. Inf. Forens. Security, vol. 6, no. 3, pp. 661-671, Sep. 2011.

[27] G. Zheng, P.-D. Arapoglou, and B. Ottersten, "Physical layer security in multibeam satellite systems," IEEE Trans. Wireless Commun., vol. 11, no. 2, pp. 852-863, Feb. 2012.

[28] Y. Yan, B. Zhang, D. Guo, S. Li, H. Niu, and X. Wang, "Joint beamforming and jamming design for secure cooperative hybrid satellite-terrestrial relay network," in Proc. IEEE 25th Wireless and Optical Communication Conference (WOCC), Chengdu, China, May 2016, pp. 1-5.

[29] C. Yuan, M. Lin, J. Ouyang, and Y. Bu, "Joint security beamforming in cognitive hybrid satellite-terrestrial networks," in Proc. IEEE 83rd
Vehicular Technology Conference (VTC-Spring), Nanjing, China, May 2016, pp. 1-5.

[30] T. A. Le, Q.-T. Vien, H. X. Nguyen, D. W. K. Ng, and R. Schober, "Robust chance-constrained optimization for power-efficient and secure SWIPT systems," IEEE Transactions on Green Communications and Networking, vol. 1, no. 3, pp. 333-346, Sep. 2017.

[31] A. Gharanjik, M. R. B. Shankar, P. D. Arapoglou, M. Bengtsson, and B. Ottersten, "Robust precoding design for multibeam downlink satellite channel with phase uncertainty," in Proc. IEEE International Conference on Acoustics, Speech and Signal Processing (ICASSP), Brisbane, Australia, Apr. 2015, pp. 3083-3087.

[32] B. Li, Z. Fei, X. Xu, and Z. Chu, "Resource allocations for secure cognitive satellite terrestrial networks," IEEE Wireless Commun. Lett., to be published.

[33] T. S. Rappaport, Wireless Communications: Principles and Practice, 2nd Edition. Prentice Hall, 2003.

[34] J. Arnau, D. Christopoulos, S. Chatzinotas, C. Mosquera, and B. Ottersten, "Performance of the multibeam satellite return link with correlated rain attenuation," IEEE Trans. Wireless Commun., vol. 13, no. 11, pp. 62866299, Nov. 2014

[35] M. Lin, J. Ouyang, and W.-P. Zhu, "Joint beamforming and power control for device-to-device communications underlaying cellular networks," IEEE J. Sel. Areas Commun., vol. 34, no. 1, pp. 138-150, Jan. 2016.

[36] S. Shi, K. An, G. Li, Z. Li, H. Zhu, and G. Zheng, "Optimal power control in cognitive satellite terrestrial networks with imperfect channel state information," IEEE Wireless Commun. Lett., to be published.

[37] M. R. A. Khandaker, K.-K. Wong, Y. Zhang, and Z. Zheng, "Probabilistically robust SWIPT for secrecy MISOME systems," IEEE Trans. Inf. Forens. Security, vol. 12, no. 1, pp. 211-226, Jan. 2017.

[38] F. Zhu and M. Yao, "Improving physical-layer security for CRNs using SINR-based cooperative beamforming," IEEE Trans. Veh. Technol. vol. 65, no. 3, pp. 1835-1841, Mar. 2016.

[39] N. Mokari, S. Parsaeefard, H. Saeedi, and P. Azmi, "Cooperative secure resource allocation in cognitive radio networks with guaranteed secrecy rate for primary users," IEEE Trans. Wireless Commun., vol. 13, no. 2, pp. 1058-1073, Feb. 2014.

[40] K. An, M. Lin, O. Jian, and T. Liang, "Outage analysis of multi-antenna cognitive hybrid satellite-terrestrial relay networks with beamforming," IEEE Commun. Lett., vol. 19, no. 7, pp. 1157-1160, Jul. 2015.

[41] T. Liang, K. An, and S. Shi, "Statistical modeling based deployment issue in cognitive satellite terrestrial networks," IEEE Wireless Commun. Lett. pp. 1157-1160, to be published.

[42] A. Nemirovski and A. Shapiro, "Convex approximations of chance constrained programs," SIAM J. Optim., vol. 17, no. 4, pp. 969-996, Nov. 2006.

[43] K.-Y. Wang, A. M.-C. So, T.-H. Chang, W.-K. Ma, and C.-Y. Chi, "Outage constrained robust transmit optimization for multiuser MISO downlinks: Tractable approximations by conic optimization," IEEE Trans. Signal Process., vol. 62, no. 21, pp. 5690-5705, Nov. 2014.

[44] S. Boyd and L. Vandenberghe, Convex Optimization. U.K.: Cambridge Univ. Press, 2004.

[45] A. Abdi, W. C. Lau, M. S. Alouini, and M. Kaveh, "A new simple model for land mobile satellite channels: First- and second-order statistics," IEEE Trans. Wireless Commun., vol. 2, no. 3, pp. 519-528, May 2003.

[46] F. Zhou, Z. Li, J. Cheng, Q. Li, and J. Si, "Robust AN-aided beamforming and power splitting design for secure MISO cognitive radio with SWIPT,' IEEE Trans. Wireless Commun., vol. 16, no. 4, pp. 2450-2464, Apr. 2017 